PROCEEDINGS OF THE

AMERICAN MATHEMATICAL SOCIETY

Volume 135, Number 1, January 2007, Pages 277-288

S 0002-9939(06)08310-9

Article electronically published on July 28, 2006

\title{
TOPOLOGY OF SPACES OF EQUIVARIANT SYMPLECTIC EMBEDDINGS
}

\author{
ALVARO PELAYO
}

(Communicated by Ronald A. Fintushel)

\begin{abstract}
We compute the homotopy type of the space of $\mathbb{T}^{n}$-equivariant symplectic embeddings from the standard $2 n$-dimensional ball of some fixed radius into a $2 n$-dimensional symplectic-toric manifold $(M, \sigma)$, and use this computation to define a $\mathbb{Z}_{\geq 0}$-valued step function on $\mathbb{R}_{\geq 0}$ which is an invariant of the symplectic-toric type of $(M, \sigma)$. We conclude with a discussion of the partially equivariant case of this result.
\end{abstract}

\section{THE MAIN THEOREM}

Let $(M, \sigma)$ be a $2 n$-dimensional symplectic manifold and write $\mathbb{B}_{r}$ for the compact $2 n$-ball of radius $r>0$ in the complex space $\mathbb{C}^{n}$ equipped with the restriction of the standard symplectic form $\sigma_{0}$ of $\mathbb{C}^{n}$. (The proofs of the results in this paper hold verbatim for the open ball.) Recently a lot of effort has been put into understanding the topological and geometric properties of the space of symplectic embeddings from $\mathbb{B}_{r}$ into $M$. This question is not only intriguing, but it is also very fundamental because it acknowledges one of the main differences that exist between Riemannian and symplectic geometry, e.g. Gromov's non-squeezing theorem [12].

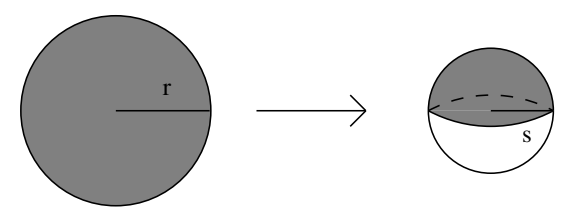

Figure 1. An equivariant and symplectic embedding $\mathbb{B}_{r}^{2} \rightarrow \mathbb{S}_{s}^{2}$ with $r / s=\sqrt{2}$.

This question, posed with such generality, has proven to be extremely difficult to answer. Significant progress has been made by McDuff [17, [18, Biran [3], [5] and most recently by Lalonde-Pinsonnault 14, among other authors. One of the most general results is due to McDuff; she showed the connectedness of the space of 4-balls into 4-manifolds with non-simple Seiberg-Witten type, in particular rational or ruled surfaces. Recall that we say that a symplectic 4-manifold $Q$ has simple Seiberg-Witten type or just simple type if the only non-zero Gromov invariants of

Received by the editors August 5, 2004 and, in revised form, April 22, 2005.

2000 Mathematics Subject Classification. Primary 53D20; Secondary 53D05.

(C)2006 American Mathematical Society 
$Q$ occur in classes $A \in \mathrm{H}_{2}(Q)$ for which $k(A)=-K \cdot A+A^{2}=0$. It follows from work of Taubes and $\mathrm{Li}$-Liu that the symplectic 4-manifolds with non-simple type are blow-ups of (i) rational and ruled manifolds; (ii) manifolds with $b_{1}=0$, $\mathrm{b}_{2}^{+}=1$, like the Enriques or Barlow surface; and (iii) manifolds with $\mathrm{b}_{1}=2$ and $\left(\mathrm{H}_{1}(Q)\right)^{2} \neq 0$; examples (with $K=0$ ) are hyperelliptic surfaces, some non-Kähler $\mathbb{T}^{2}$-bundles over $\mathbb{T}^{2}$ and quotients $\mathbb{T}^{2} \times \Sigma_{g} / G$ where $\Sigma_{g}$ is a surface of genus $g$ greater than 1 , and $G$ is certain finite group. See 17 for further references and examples.

McDuff's techniques are unique to dimension 4 and do not extend at all to higher dimensions - this is also the case in the other authors' work - existence of $J$ holomorphic curves with special homological properties is essential in their proofs. Although $J$-holomorphic curves exist in all even dimensions, it is only in dimension 4 where these homological properties hold.

In the present paper we study a special case of this question: $M$ is a symplectictoric manifold of arbitrary dimension, and the symplectic embeddings that we consider preserve the toric structure, see Figure 1. Precisely this means that there exists an automorphism $\Lambda$ of the $n$-torus $\mathbb{T}^{n}$ such that the following diagram commutes:

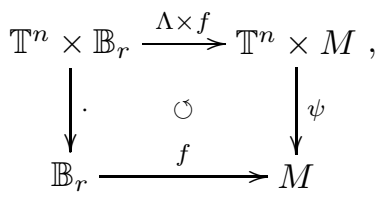

where $\psi$ is a fixed effective and Hamiltonian $\mathbb{T}^{n}$-action on $M$ and $\cdot$ denotes the standard action by rotations on $\mathbb{B}_{r}$ (component by component). In this case we say that $f$ is a $\Lambda$-equivariant mapping.
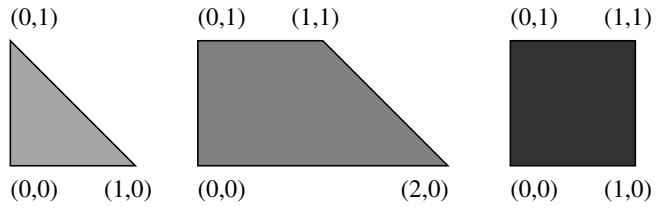

Figure 2. The momentum polytope of $\mathbb{C P}^{2}$ and $\mathbb{B}_{1}^{2}$ (left), of a Hirzebruch surface (center) and of $\left(\mathbb{C P}^{1}\right)^{2}$ (right).

The main feature that makes the study of symplectic manifolds equipped with torus actions richer than the study of generic symplectic manifolds is the existence of the momentum map $\mu^{M}: M \rightarrow \operatorname{Lie}\left(\mathbb{T}^{n}\right)^{*}$ whose image $\Delta^{M}$ is a convex polytope (called the momentum polytope of $M$, cf. Figure 2) as shown independently by Atiyah and Guillemin-Sternberg [1, 8]. Here we are identifying the Lie algebra $\operatorname{Lie}\left(\mathbb{T}^{n}\right)$ and its dual $\operatorname{Lie}\left(\mathbb{T}^{n}\right)^{*}$ with $\mathbb{R}^{n}$. Since this identification is not canonical, we need to specify the convention we adopt in this paper. This amounts to choosing an epimorphism $\mathbb{R} \rightarrow \mathbb{T}^{1}$ which we take to be $x \mapsto e^{2 \sqrt{-1} x}$. This epimorphism induces an isomorphism between $\operatorname{Lie}\left(\mathbb{T}^{1}\right)$ and $\mathbb{R}$ via $\frac{\partial}{\partial x} \mapsto 1 / 2$, giving rise to a new isomorphism Lie $\left(\mathbb{T}^{n}\right) \rightarrow \mathbb{R}^{n}, \frac{\partial}{\partial x_{k}} \mapsto 1 / 2 e_{k}$, by canonically identifying $\operatorname{Lie}\left(\mathbb{T}^{n}\right)$ with the product of $n$ copies of $\operatorname{Lie}\left(\mathbb{T}^{1}\right)$ (see [9] for more details).

For example, under the convention of the previous paragraph, the momentum map $\mu^{\mathbb{B}_{r}}$ of $\mathbb{B}_{r}$ is a mapping from $\mathbb{B}_{r}$ into $\mathbb{R}^{n}$ with components $\mu_{k}^{\mathbb{B}_{r}}(z)=\left|z_{k}\right|^{2}$, for all 
integer $k$ with $1 \leq k \leq n$. (There are a number of different conventions used in the literature, and our choice is intended to give the simplest formula for the momentum map of $\mathbb{B}_{r}$.) The simplest symplectic manifolds which admit Hamiltonian effective torus actions are called symplectic-toric.

Definition 1.1. A symplectic-toric manifold $M$, also called a Delzant manifold, is a compact connected symplectic manifold equipped with an effective Hamiltonian action of a torus of dimension half of the dimension of the manifold. In this case the momentum polytope $\Delta^{M}$ is called the Delzant polytope of $M$.

Symplectic-toric manifolds were classified by Delzant in [7]. In particular, he showed that the momentum image of such a manifold under the momentum map completely determines $M$ up to equivariant symplectomorphism.

The main result of this paper, Theorem 1.1 below, describes the topology of the space of equivariant embeddings of symplectic balls into a symplectic-toric manifold. We denote by $\chi(M)$ the Euler characteristic of $M$.

Theorem 1.1. For every symplectic-toric $2 n$-manifold $M$ there is an associated $\mathbb{Z}$-valued non-increasing step function $\operatorname{Emb}_{(M, \sigma)}: \mathbb{R}_{\geq 0} \rightarrow[0, n ! \chi(M)]$ such that for each $r \geq 0$ the space of equivariant symplectic embeddings from the $2 n$-ball $\mathbb{B}_{r}$ into $M$ is homotopically equivalent to a disjoint union of $\operatorname{Emb}_{(M, \sigma)}(r)$ subspaces, each of which is homeomorphic to the $n$-torus $\mathbb{T}^{n}$. $\Delta^{M}$ :

As a matter of fact we can explicitly and easily read $\operatorname{Emb}_{(M, \sigma)}$ from the polytope

Example 1. Let $(M, \sigma)$ equal the blow-up of $\mathbb{S}_{r_{0}}^{2} \times \mathbb{S}_{r_{0}}^{2}$ with $r_{0}=1 / \sqrt{2}$ whose Delzant polytope has vertices at $(0,0),(2,0),(2,1),(1,2)$ and $(0,2)$ (see Figure 4$)$. Then $\operatorname{Emb}_{(M, \sigma)}=10 \chi_{[0,1)}+2 \chi_{[1, \sqrt{2})}$, where $\chi_{A}$ denotes the characteristic function of $A \subset \mathbb{R}$. We identify the 2 -sphere of radius $r$ equipped with the standard area form with $\left(\mathbb{C P}^{1}, 4 r^{2} \cdot \sigma_{\mathrm{FS}}\right)$, where $\sigma_{\mathrm{FS}}$ is the Fubini-Study form.

Proposition 1.1. The function $\operatorname{Emb}_{(M, \sigma)}(r)$ given in Theorem 1.1 is an invariant of the symplectic-toric type of $M$ and is given by the formula

$$
\operatorname{Emb}_{(M, \sigma)}(r)=n ! \sum_{p \in M^{\mathbb{T}^{n}}} \mathrm{c}_{p}(r),
$$

where for each fixed point $p \in M, \mathrm{c}_{p}(r)=1$ if the infimum of the $\operatorname{SL}(n, \mathbb{Z})$-lengths of the edges of $\Delta^{M}$ meeting at $\mu^{M}(p)$ is strictly greater than $r^{2}$, and $\mathrm{c}_{p}(r)=0$ otherwise.

Example 2. Let $\sigma_{\mathrm{FS}}$ be the Fubini-Study form on $\mathbb{C P}^{n}$ and observe that $\mathbb{T}^{n}$ acts naturally on $\left(\mathbb{C P}^{n}, \lambda \cdot \sigma_{\mathrm{FS}}\right), \lambda>0$, with $n+1$ fixed points. The momentum polytope is a tetrahedrum with vertices at 0 and $\lambda e_{i}$, where the $e_{i}$ are the canonical basis vectors in $\mathbb{R}^{n}$. So if $M=\mathbb{C P}^{n} \times \mathbb{C P}^{m}$, the space of equivariant symplectic embeddings from $\mathbb{B}_{r}$ into $M$ is homotopically equivalent to

$$
\bigsqcup_{k=1}^{(n+m) !(n+1)(m+1)} \mathbb{T}^{n+m}
$$

if $r<\sqrt{\lambda}$, and it is empty otherwise. 
The study of the space of symplectic embeddings is directly related to the study of the symplectic ball packing problem cf. [4], the equivariant version of which was treated in [19].

\section{Proof of Theorem 1.1}

In this section we prove Theorem 1.1 For clarity, the proof is divided in three steps, which we describe next. We start by introducing the notation and making the following observations:

i) Throughout the proof $\mathcal{R}$ will denote the space of rotations by matrices of the form $\left(\delta_{i \tau(i)} \theta_{i j}\right)_{i, j=1}^{n}$ with $\tau \in \mathrm{S}_{n}$ (the symmetric group) and $\theta_{i j} \in \mathbb{T}^{1}$, and $\mathcal{E}_{x}^{\mathbb{T}^{n}}$ will denote the space of equivariant symplectic embeddings $f$ from $\mathbb{B}_{r}$ into $M$ such that $f(0)=p$ and $\mu^{M}(p)=x \in \Delta^{M}$, equipped with the $\mathrm{C}^{m}$-Whitney topology $(m \geq 0)$. Throughout the present section we fix $f$.

Since each component of $\mathcal{R}$ is canonically identified with the $n$-torus $\mathbb{T}^{n}$ (cf. Corollary 2.1), Theorem 1.1 amounts to prove that if $p \in M^{\mathbb{T}^{n}}\left(M^{\mathbb{T}^{n}}\right.$ denotes the $\mathbb{T}^{n}$-fixed point set) is such that $\mu^{M}(p)=x$, then the space $\mathcal{E}_{x}^{\mathbb{T}^{n}}$ gets identified with $\mathcal{R}$ via a homotopy equivalence.

ii) Secondly let $\mathcal{B}_{r}^{\mathbb{T}^{n}}$ denote the space of equivariant symplectomorphisms of $\mathbb{B}_{r}$ (again with respect to the $\mathrm{C}^{m}$-Whitney topology).

Recall that, for example, the $\mathrm{C}^{m}$-Whitney topology on $\mathcal{B}_{r}^{\mathbb{T}^{n}}$ is given by the well-known norm

$$
\|\phi\|_{\mathrm{C}^{m}}=\max _{0 \leq k \leq m} \sup _{z \in \mathbb{B}_{r}}\left\|\mathrm{~T}^{k} \phi\right\|_{\mathfrak{M}(\mathbb{C})},
$$

where we are taking the norm $\|\cdot\|_{\mathfrak{M}(\mathbb{C})}$ on the right-hand side of this expression to be the canonical Euclidean norm on the space of $n \times n$ matrices with complex entries.

iii) We identify the automorphism group $\operatorname{Aut}\left(\mathbb{T}^{n}\right)$ with the matrix group $\mathrm{GL}(n, \mathbb{Z})$.

iv) The elements $\alpha_{i}^{p}$ 's, $1 \leq i \leq n$, denote the weights of the isotropy representation of $\mathbb{T}^{n}$ on $\mathrm{T}_{p} M$; the canonical basis vectors $e_{i} \in \mathbb{R}^{n}$ represent the weights of the isotropy representation of $\mathbb{T}^{n}$ on $\mathrm{T}_{0} \mathbb{B}_{r}$.

Step 1: Invariance of the image $f\left(\mathbb{B}_{r}\right)$.

In this step we first show how to go from smooth maps on manifolds to affine maps on polytopes (see diagram (2.3) ), and secondly we use this to show the invariance of the image $f\left(\mathbb{B}_{r}\right) \subset M$. Precisely, one can think of an embedding being equivariant in the sense of commuting with the $\mathbb{T}^{n}$-action, and it is when we reparametrize the torus that $\Lambda$ appears.

Lemma 2.1. Let $g$ be any $\Lambda$-equivariant and symplectic embedding such that the normalization condition $f(0)=g(0)=p$ holds. Then for all $z \in \mathbb{B}_{r}$, if $\mathbb{T}^{n} \cdot z$ denotes the $\mathbb{T}^{n}$-orbit that passes through $z$, the identity $f\left(\mathbb{T}^{n} \cdot z\right)=g\left(\mathbb{T}^{n} \cdot z\right)$ holds, and therefore $f\left(\mathbb{B}_{r}\right)=g\left(\mathbb{B}_{r}\right)$.

Proof. Let $f, g$ be $\Lambda$-equivariant and symplectic embeddings from $\mathbb{B}_{r}$ into $M$ with $f(0)=g(0)=p$. Under the identifications described in Section 1, the following diagram commutes, where the top arrow stands for the affine map with linear part 
$\left(\Lambda^{\mathrm{t}}\right)^{-1}$, which takes 0 to $x$ :

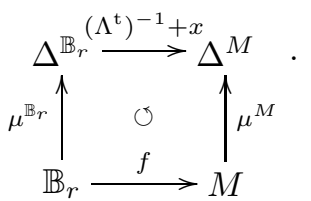

In order to prove the commutativity of diagram (2.3), we denote by $\xi_{M}$ the vector field induced by the element $\xi \in \operatorname{Lie}\left(\mathbb{T}^{n}\right)$ via the exponential map and note that from the definition of the momentum maps $\mu^{M}$ and $\mu^{\mathbb{B}_{r}}$, the $\Lambda$-equivariance of $f$, and the fact that $f^{*} \sigma=\sigma_{0}$, we have the following sequence of equalities, where $\mathrm{T}_{f(z)} \mu^{M}$ and $\mathrm{T}_{z} \mu^{\mathbb{B}_{r}}$ denote, respectively, the tangent mapping of $\mu^{M}$ at $f(z)$ and of $\mu^{\mathbb{B}_{r}}$ at $z$,

$$
\begin{aligned}
\left\langle\mathrm{T}_{z} \mu^{\mathbb{B}_{r}}(v), \xi\right\rangle_{z} & =\left(\sigma_{0}\right)_{z}\left(v, \xi_{\mathbb{B}_{r}}(z)\right) \\
& =\sigma_{f(z)}\left(\mathrm{T}_{z} f(v), \Lambda(\xi)_{M}(f(z))\right) \\
& =\left\langle\mathrm{T}_{f(z)} \mu^{M}\left(\mathrm{~T}_{z} f(v)\right), \Lambda(\xi)\right\rangle_{f(z)} \\
& =\left\langle\Lambda^{\mathrm{t}} \circ \mathrm{T}_{f(z)} \mu^{M}\left(\mathrm{~T}_{z} f(v)\right), \xi\right\rangle_{z},
\end{aligned}
$$

where $z \in \mathbb{B}_{r}, v \in \mathrm{T}_{z} \mathbb{B}_{r}$ and $\xi \in \operatorname{Lie}\left(\mathbb{T}^{n}\right)$. Therefore by equation (2.4) and by using the chain rule we obtain that for all $z \in \mathbb{B}_{r}$ and $v \in \mathrm{T}_{z} \mathbb{B}_{r}$

$$
\mathrm{T}_{z} \mu^{\mathbb{B}_{r}}(v)=\mathrm{T}_{z}\left(\Lambda^{\mathrm{t}} \circ \mu^{M} \circ f\right)(v) .
$$

Considering equation (2.5), using the fact that $\mathbb{T}^{n}$ is a connected group, $f(0)=$ $p$ and $\mu^{M}(p)=x$ and composing with $\left(\Lambda^{\mathrm{t}}\right)^{-1}$, after integration we obtain the commutativity condition on diagram (2.3). Notice that diagram (2.3) also holds for the embedding $g$.

Then it follows from the conjunction of diagram (2.3) and diagram (1.1) that for all $t \in \mathbb{T}^{n}$ the following identities hold:

$$
\begin{aligned}
\mu^{M}(\psi(\Lambda(t), f(z))) & =\mu^{M}(f(t \cdot z)) \\
& =\left(\Lambda^{\mathrm{t}}\right)^{-1} \circ \mu^{\mathbb{B}_{r}}(z)+x \\
& =\mu^{M}(g(t \cdot z))=\mu^{M}(\psi(\Lambda(t), g(z))) .
\end{aligned}
$$

Expression (2.6) is clearly equivalent to $\mu^{M}\left(\mathbb{T}^{n} \cdot f(z)\right)=\mu^{M}\left(\mathbb{T}^{n} \cdot g(z)\right)$, since $\Lambda$ is an automorphism. Now since $M$ is symplectic-toric, by the proof of the AtiyahGuillemin-Sternberg convexity theorem we know that each fiber of the momentum map $\mu^{M}$ consists of a single connected orbit which together with the last equality implies that $\mathbb{T}^{n} \cdot f(z)=\mathbb{T}^{n} \cdot g(z)$. Since $f\left(\mathbb{B}_{r}\right)$ is the union of the orbit images $f\left(\mathbb{T}^{n} \cdot z\right)$, we immediately obtain that $f\left(\mathbb{B}_{r}\right)=g\left(\mathbb{B}_{r}\right)$, which concludes the proof.

It is possible to explicitly describe the momentum image $\mu^{M}\left(f\left(\mathbb{B}_{r}\right)\right)$, and for this purpose we recall the notion of $\operatorname{SL}(n, \mathbb{Z})$-length: if $x, y \in \mathbb{R}^{n}$, we say that a segment line $[x, y]$ joining $x$ to $y$ in $\mathbb{R}^{n}$ has $\mathrm{SL}(n, \mathbb{Z})$-length $d$ if there exists a matrix $A \in \mathrm{SL}(n, \mathbb{Z})$ such that $A\left(d e_{1}\right)=y-x$ ( $d$ is not defined in general, only for segments of rational slope). The Euclidean length of a segment line agrees with its $\operatorname{SL}(n, \mathbb{Z})$-length if and only if the segment is parallel to one of the coordinate axes in $\mathbb{R}^{n}$.

In their article [16, Karshon and Tolman made the following two definitions. Let $\left(Q, \sigma^{Q}\right)$ be a connected symplectic $2 m$-dimensional manifold with momentum 

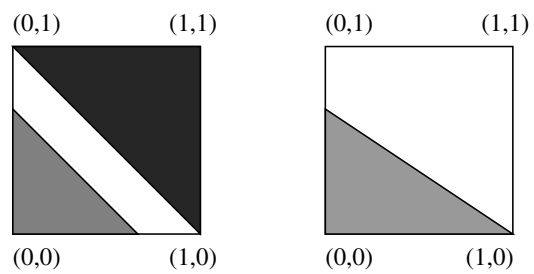

Figure 3. Equivariant symplectic ball embeddings in $\left(\mathbb{C P}^{1}\right)^{2}$ (left); the triangle on the right does not come from such embedding.

map $\mu^{Q}$ for an action of an $m$-torus $\mathbb{T}^{m}$ on $Q$, and let $\Gamma \subset\left(\operatorname{Lie}\left(\mathbb{T}^{m}\right)\right)^{*}$ be an open convex subset which contains the image of $Q$ under the momentum map $\mu^{Q}$. The quadruple $\left(Q, \sigma^{Q}, \mu^{Q}, \Gamma\right)$ is a proper Hamiltonian $\mathbb{T}^{m}$ - manifold if the momentum map $\mu^{Q}$ is proper as a map to $\Gamma$.

The proper Hamiltonian $\mathbb{T}^{m}$-manifold $\left(Q, \sigma, \mu^{Q}, \Gamma\right)$ is said to be centered about a point $\alpha \in \Gamma$ if $\alpha$ is contained in the momentum map image of every component of $Q^{K}$, for each $K \subset \mathbb{T}^{m}$. Here

$$
Q^{K}:=\left\{q \in Q \mid \psi_{Q}(a, q)=q, \forall a \in K\right\},
$$

where $\psi_{Q}: \mathbb{T}^{m} \times Q \rightarrow Q$ denotes the action of $\mathbb{T}^{m}$ on $Q$. The following lemma is Proposition 2.8 in [16].

Lemma 2.2 (Karshon-Tolman, 16]). Let the quadruple $\left(Q, \sigma^{Q}, \mu^{Q}, \Gamma\right)$ be a proper Hamiltonian $2 m$-dimensional $\mathbb{T}^{m}$-manifold. Suppose that $\left(Q, \sigma^{Q}, \mu^{Q}, \Gamma\right)$ is centered about $\alpha \in \Gamma$ and that the preimage $\left(\mu^{Q}\right)^{-1}(\{\alpha\})$ consists of a single fixed point $q$. Then $Q$ is equivariantly symplectomorphic to

$$
\left\{\left.z \in \mathbb{C}^{m}\left|\alpha+\sum_{j=1}^{m}\right| z_{j}\right|^{2} \eta_{j}^{q} \in \Gamma\right\},
$$

where $\eta_{1}^{q}, \ldots, \eta_{m}^{q}$ are the weights of the isotropy representation of $\mathbb{T}^{m}$ on $\mathrm{T}_{q} Q$.

We use Lemma 2.2 in order to prove the following lemma.

Lemma 2.3. The momentum image $\mu^{M}\left(f\left(\mathbb{B}_{r}\right)\right)$ equals the subset of $\mathbb{R}^{n}$ given by the convex hull of $x$ and $x+r^{2} \alpha_{i}^{p}$, where $1 \leq i \leq n$. Furthermore, the infimum of the $\mathrm{SL}(n, \mathbb{Z})$-lengths of the edges of $\Delta^{M}$ meeting at $x$ is greater than or equal to $r^{2}$, if and only if for all $0<s<r$ there exists an embedding $h: \mathbb{B}_{s} \rightarrow M$ which is $\Lambda$-equivariant and symplectic, satisfying $h(0)=p$.

Proof. The first observation is that $\Delta^{\mathbb{B}_{r}}$ equals the convex hull in $\mathbb{R}^{n}$ of 0 and $r^{2} e_{1}, \ldots, r^{2} e_{n}$. Secondly, since $\mu^{\mathbb{B}_{r}}: \mathbb{B}_{r} \rightarrow \Delta^{\mathbb{B}_{r}}$ is onto, it follows from diagram (2.3) that

$$
\mu^{M}\left(f\left(\mathbb{B}_{r}\right)\right)=\left(\Lambda^{\mathrm{t}}\right)^{-1}\left(\Delta^{\mathbb{B}_{r}}\right)+x .
$$

Since $\Lambda$ is an automorphism, $\left(\Lambda^{\mathrm{t}}\right)^{-1}$ is an automorphism of the corresponding dual spaces and therefore there exists a permutation $\tau \in \mathrm{S}_{n}$ such that $\left(\Lambda^{\mathrm{t}}\right)^{-1}\left(e_{i}\right)=\alpha_{\tau(i)}^{p}$. Then the linearity of $\Lambda$ implies that $\mu^{M}\left(f\left(\mathbb{B}_{r}\right)\right)$ equals the the convex hull in $\mathbb{R}^{n}$ of the points $x$ and $x+r^{2} \alpha_{i}^{p}, 1 \leq i \leq n$, which proves the first claim.

Suppose that the infimum of the $\operatorname{SL}(n, \mathbb{Z})$-lengths of the edges meeting at $x$ is greater than or equal to $r^{2}$. Let $\Sigma$ be the convex hull of $x$ and $x+r^{2} \alpha_{i}^{p}$, with 
$1 \leq i \leq n$, and let $Z$ be the convex hull of $x+r^{2} \alpha_{i}^{p}$, with $1 \leq i \leq n$. Notice that $\Sigma \subset \Delta^{M}, \Sigma \backslash Z$ is open in $\Delta^{M}$, and let $\Gamma \subset \mathbb{R}^{n}$ be the open half-space of $\mathbb{R}^{n}$, whose closure's boundary $\partial(\operatorname{cl}(\Gamma))$ is the hyperplane of $\mathbb{R}^{n}$ that contains $Z$, and such that $\Sigma \backslash Z \subset \Gamma$.

Let $N:=\left(\mu^{M}\right)^{-1}(\Sigma \backslash Z)$ and let $\sigma^{N}$ be the symplectic form obtained by restrict$\operatorname{ing} \sigma$ to $N$. The set $N$ is open in $M$ because it is the preimage of the open set $\Sigma \backslash Z$ under the momentum map $\mu^{M}: M \rightarrow \Delta^{M}$. By the proof of Atiyah-GuilleminSternberg convexity theorem, cf. [1], [8, $N$ is a connected manifold. Since $M$ is compact, the momentum map $\mu^{M}: M \rightarrow \Delta^{M}$ is a proper map and therefore its restriction $\mu^{M}: N \rightarrow \Sigma \backslash Z$ is a proper map, which means that $\mu^{M}: N \rightarrow \Gamma$ is proper, since $\left(\mu^{M}\right)^{-1}(\Gamma \backslash(\Sigma \backslash Z))=\emptyset$. Therefore $\left(N, \sigma^{N}, \psi^{N}\right)$ is a connected symplectic manifold with momentum map $\mu^{M}$, and the quadruple $\left(N, \sigma^{N}, \mu^{M}, \Gamma\right)$ is a proper Hamiltonian $\mathbb{T}^{n}$-space.

On the other hand, notice that the quadruple $\left(N, \sigma^{N}, \mu^{M}, \Gamma\right)$ is centered about the point $x$, and $\left(\mu^{M}\right)^{-1}(\{x\})=p$, so we can apply Lemma 2.2. and conclude that $N$ is equivariantly symplectomorphic to the submanifold $X \subset \mathbb{C}^{n}$ given by

$$
\begin{aligned}
X & :=\left\{\left.z \in \mathbb{C}^{n}\left|x+\sum_{i=1}^{n}\right| z_{i}\right|^{2} \alpha_{i}^{p} \in \Sigma \backslash Z\right\} \\
& =\left\{\left.z \in \mathbb{C}^{n}\left|x+\sum_{i=1}^{n}\right| z_{i}\right|^{2} \alpha_{i}^{p} \in \Sigma\right\} \backslash\left\{\left.z \in \mathbb{C}^{n}\left|x+\sum_{i=1}^{n}\right| z_{i}\right|^{2} \alpha_{i}^{p} \in Z\right\} \\
& =\mathbb{B}_{r} \backslash \partial \mathbb{B}_{r}=\operatorname{Int}\left(\mathbb{B}_{r}\right) .
\end{aligned}
$$

Hence there exists an equivariant symplectomorphism $\phi: \operatorname{Int}\left(\mathbb{B}_{r}\right) \rightarrow N$, and by letting $j: \mathbb{B}_{s} \rightarrow \operatorname{Int}\left(\mathbb{B}_{r}\right)$ be the standard inclusion, if $s<r$, the map $h:=j_{N} \circ \phi \circ j:$ $\mathbb{B}_{s} \rightarrow M$, where $j_{N}: N \rightarrow M$ is the inclusion map, is an equivariant symplectic embedding for all $s<r$ with $h(0)=p$. The converse follows from the first statement of the lemma.

Note that $\mu^{M}\left(f\left(\mathbb{B}_{r}\right)\right)$ only depends on the fixed point $p$ and the radius $r$ (which was fixed a priori) and not on $f$. In Figure 3 several momentum ball images are drawn using Lemma 2.3. Note that the shaded triangle on the right picture is not a Delzant polytope since it fails to be smooth at $(0,0)$. Delzant polytopes are simple, edge-rational and smooth polytopes, cf. Figure 2 (see [10] or [6] for a definition of these notions).

Step 2: A deformation retraction on $\mathcal{B}_{r}^{\mathbb{T}^{n}}$.

In this step we use Alexander's trick to construct a deformation retraction from the space of equivariant symplectomorphisms of the $2 n$-dimensional ball $\mathbb{B}_{r}$ in $\mathbb{C}^{n}$ onto a disjoint union of copies of $\mathbb{T}^{n}$. The continuity of this deformation is standard and may be found in 13 .

Lemma 2.4. The space $\mathcal{B}_{r}^{\mathbb{T}^{n}}$ of equivariant symplectomorphisms of the $2 n$-dimensional ball $\mathbb{B}_{r}$ in $\mathbb{C}^{n}$, with respect to the standard symplectic form $\sigma_{0}$ and the canonical action of $\mathbb{T}^{n}$ by rotations, deformation retracts onto its subspace of linear, equivariant and symplectic rotations given by matrices in $\mathcal{R}$.

Proof. We define the transformation $H_{\mathbb{B}_{r}}^{\mathbb{T}^{n}}$ from $\mathcal{B}_{r}^{\mathbb{T}^{n}} \times[0,1]$ into $\mathcal{B}_{r}^{\mathbb{T}^{n}}$, by the formula $H_{\mathbb{B}_{r}}^{\mathbb{T}^{n}}(\phi, t):=\phi_{t}$, where $\phi_{t}$ is the composite map

$$
\phi_{t}:=\left(m_{t}\right)^{-1} \circ \phi \circ m_{t}, t \neq 0 .
$$


The map $m_{t}$ in expression (2.7) denotes the linear contraction of factor $0 \leq t \leq 1$ on $\mathbb{B}_{r}, m_{t}(z)=t z$; and when $t=0, \phi_{t}=\phi_{0}$ is defined to be the tangent mapping $\mathrm{T} \phi$ of the map $\phi$, evaluated at 0 . (This expression for $\phi_{t}$ is known as Alexander's trick.) It is easy to check that $H_{\mathbb{B}_{r}}^{\mathbb{T}^{n}}$ is continuous and that the evaluation map $[0,1] \times \mathbb{B}_{r} \rightarrow \mathbb{B}_{r}$ given by $(t, z) \mapsto \phi_{t}(z)$ is smooth. Since $H_{\mathbb{B}_{r}}^{\mathbb{T}^{n}}$ is the identity on linear maps, we conclude that it is a deformation retraction, not only a homotopy, onto the space of ball rotations by matrices $\left(\delta_{i \tau(i)} \theta_{i j}\right)_{i, j=1}^{n}$ with $\tau \in \mathrm{S}_{n}$ (the symmetric group) and $\theta_{i j} \in \mathbb{T}^{1}$.

We have left to check that $H_{\mathbb{B}_{r}}^{\mathbb{T}^{n}}$ is well defined, i.e. that $\phi_{t} \in \mathcal{B}_{r}^{\mathbb{T}^{n}}$. Indeed, the equivariance of the mapping $\phi_{t}$ follows directly from formula (2.7); explicitely we have that if $\phi$ is equivariant with respect to $\Lambda \in \operatorname{Aut}\left(\mathbb{T}^{n}\right)$, then $\phi_{t}(s \cdot z)=$ $1 / t \phi(s \cdot t z)=\Lambda(s) \cdot \phi_{t}(z)$ for all $s \in \mathbb{T}^{n}$. By differentiating formula (2.7) we obtain that

$$
\mathrm{T}\left(\phi_{t}\right)=\left(m_{t}\right)^{-1} \circ \mathrm{T} \phi \circ m_{t},
$$

and since $m_{t}$ is a linear isomorphism, the mapping $\phi_{t}$ is a diffeomorphism. Furthermore, since the mapping $\phi$ is symplectic, it follows from expression (2.8) that for all $z \in \mathbb{B}_{r}$ we have that $\left(\phi_{t}^{*} \sigma_{0}\right)_{z}(u, v)=\left(\sigma_{0}\right)_{\phi(z)}\left(\mathrm{T}_{z} \phi(u), \mathrm{T}_{z} \phi(v)\right)=\left(\sigma_{0}\right)_{z}(u, v)$, for every pair of vectors $u, v \in \mathrm{T}_{z} \mathbb{B}_{r}$, and hence $\phi_{t}$ is a symplectic mapping. Therefore $\phi_{t}$ is a diffeomorphism, which is equivariant and symplectic, or equivalently $\phi_{t} \in \mathcal{B}_{r}^{\mathbb{T}^{n}}$. We have been assuming that $t \neq 0$, but if $t=0$, it is trivial that $\phi_{0} \in \mathcal{B}_{r}^{\mathbb{T}^{n}}$.

Corollary 2.1. The space $\mathcal{B}_{r}^{\mathbb{T}^{n}}$ of equivariant symplectomorphisms of the $2 n$ dimensional ball $\mathbb{B}_{r}$ in $\mathbb{C}^{n}$, with respect to the standard symplectic form $\sigma_{0}$ and the canonical action of $\mathbb{T}^{n}$ by rotations, is homotopically equivalent to a disjoint union of $n$ ! copies of $\mathbb{T}^{n}$.

Proof. Apply Lemma 2.4 and observe that the space of ball rotations by matrices $\left(\delta_{i \tau(i)} \theta_{i j}\right)_{i, j=1}^{n}$ with $\tau \in \mathrm{S}_{n}$ and $\theta_{i j} \in \mathbb{T}^{1}$ is homotopically equivalent to a disjoint union of $n$ ! copies of $\mathbb{T}^{n}$.

We conclude the proof with Step 3, in which Lemma 2.1, Lemma 2.3 and Lemma 2.4 are combined in order to prove Theorem 1.1. The proof of Proposition [1.1 will follow from the proof of Theorem 1.1, since the function $\operatorname{Emb}_{(M, \sigma)}$ will be explicitly computed.

Step 3: Lifting the deformation $\phi_{t}$ to $\mathcal{E}_{x}^{\mathbb{T}^{n}}$ and conclusion.

In this final step we show that $\mathcal{E}_{x}^{\mathbb{T}^{n}}$ is homotopically equivalent to a disjoint union of copies of $\mathbb{T}^{n}$.

Lemma 2.5. Suppose that the infimum of the $\mathrm{SL}(n, \mathbb{Z})$-lengths of the edges of $\Delta^{M}$ meeting at $x$ is strictly greater than $r^{2}$. Then there exists an equivariant and symplectic embedding $u: \mathbb{B}_{r} \rightarrow M$ with $u(0)=p$ such that if $\rho$ is the identification map on $\mathcal{E}_{x}^{\mathbb{T}^{n}}$ which takes values on $\mathcal{B}_{r}^{\mathbb{T}^{n}}$ and is given by formula $\rho(h):=u^{-1} \circ h$, where $h \in \mathcal{E}_{x}^{\mathbb{T}^{n}}$, the space $\mathcal{E}_{x}^{\mathbb{T}^{n}}$ is homotopically equivalent to the space $\rho^{-1}(\mathcal{R})$.

Proof. The first observation is that by Lemma 2.3 there exists a $\Lambda$-equivariant and symplectic embedding $u$ from $\mathbb{B}_{r}$ into $M$ with $u(0)=p$. In order to construct homotopy equivalences between $\mathcal{E}_{x}^{\mathbb{T}^{n}}$ and $\mathcal{R}$, we define $\rho$ to be the identification map on $\mathcal{E}_{x}^{\mathbb{T}^{n}}$ which takes values in $\mathcal{B}_{r}^{\mathbb{T}^{n}}$ and is given by formula $\rho(h):=u^{-1} \circ h$ for 
every $h \in \mathcal{E}_{x}^{\mathbb{T}^{n}}$. Now we claim that the map $H_{x}^{\mathbb{T}^{n}}$ from $\mathcal{E}_{x}^{\mathbb{T}^{n}} \times[0,1]$ to $\mathcal{E}_{x}^{\mathbb{T}^{n}}$, given by the commutative diagram (2.9) below, is a well-defined and continuous homotopy satisfying $H_{x}^{\mathbb{T}^{n}}\left(\mathcal{E}_{x}^{\mathbb{T}^{n}} \times\{0\}\right)=\rho^{-1}(\mathcal{R})$, while $\mathcal{E}_{x}^{\mathbb{T}^{n}}$ is preserved at time $t=1$, i.e. we have that $H_{x}^{\mathbb{T}^{n}}\left(\mathcal{E}_{x}^{\mathbb{T}^{n}} \times\{1\}\right)=\mathcal{E}_{x}^{\mathbb{T}^{n}}$. The diagram is the following:

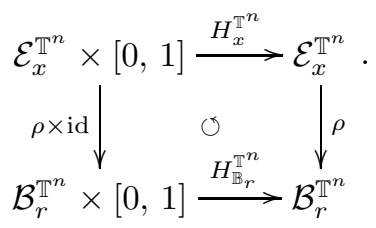

The mapping $H_{x}^{\mathbb{T}^{n}}$ is well defined by Lemma2.1. Note that $H_{x}^{\mathbb{T}^{n}}$ is continuous, since the identifications $\rho$ and $\rho^{-1}$ are obviously continuous and we showed in Lemma 2.4 that $H_{\mathbb{B}_{r}}^{\mathbb{T}^{n}}$ is continuous. We can therefore conclude, from the previous considerations and the fact that that $H_{\mathbb{B}_{r}}^{\mathbb{T}^{n}}$ is a deformation retraction in the $\mathrm{C}^{m}$-Whitney topology, that $H_{x}^{\mathbb{T}^{n}}$ induces homotopy equivalences $\rho$ and $\delta(f):=H_{x}^{\mathbb{T}^{n}}(f, 0)$ between $\mathcal{E}_{x}^{\mathbb{T}^{n}}$ and $\rho^{-1}(\mathcal{R})$, with $\rho \circ \delta$ homotopic to $\operatorname{id}_{\mathcal{E}_{x}^{\pi^{n}}}$ and $\delta \circ \rho=\operatorname{id}_{\rho^{-1}(\mathcal{R})}$.

In order to conclude the proof of Theorem 1.1 we simply make the following observations:

- First, the space described in it is precisely the disjoint union of the $\mathcal{E}_{x}^{\mathbb{T}^{n}}, x$ being a vertex of $\Delta^{M}$, because 0 is to be mapped to a fixed point of $\psi$.

- The number of $\mathbb{T}^{n}$-fixed points, which is the same as the number of vertices of $\Delta^{M}$, is precisely $\chi(M)$. This follows from the analysis of the momentum map as in Atiyah-Delzant-Guillemin-Sternberg theory (see for example [10], 11]).

- If we denote by $\operatorname{Emb}_{(M, \sigma)}(r)$ the number of copies of $\mathbb{T}^{n}$ onto which the space considered in Theorem 1.1 retracts (see formula (1.2) ), $\operatorname{Emb}_{(M, \sigma)}(r)$ is obtained by multiplying the number of fixed points that admit such an embedding (see Lemma 2.3) by the number of copies of $\mathbb{T}^{n}$ onto which $\mathcal{E}_{x}^{\mathbb{T}^{n}}$ (for the particular point) retracts; this latter number is $n$ ! (see Corollary 2.1), i.e. as many copies of $\mathbb{T}^{n}$ as possible ways that the canonical basis vectors $e_{i}$ may be mapped onto the basis of weights $\alpha_{i}^{p}$ (for the particular point). Also, the former number is by Lemma 2.3 controlled by the Boolean variable $\mathrm{c}_{p}(r)$ defined in Proposition 1.1. Therefore $\operatorname{Emb}_{(M, \sigma)}(r)$ is given by $\operatorname{Emb}_{(M, \sigma)}(r)=n ! \sum_{p \in M^{\mathbb{T}^{n}}} \mathrm{c}_{p}(r)$, as we wanted to show.

- It is obviously true that if $(M, \sigma)$ is equivariantly symplectomorphic to $(\widetilde{M}, \widetilde{\sigma})$, then $\operatorname{Emb}_{(M, \sigma)}(r)=\operatorname{Emb}_{(\widetilde{M}, \widetilde{\sigma})}(r)$, so the integer $\operatorname{Emb}_{(M, \sigma)}(r)$ is a symplectic-toric invariant.

As a final remark we observe that the invariant function $\operatorname{Emb}_{(M, \sigma)}$ associated to the Delzant manifold $(M, \sigma)$ always reaches its minimum and maximum values on an interval of strictly positive length.

Lemma 2.6. There exist numbers $r_{0}, s_{0}>0$ such that if $r \leq r_{0}$, then the space of equivariant symplectic embeddings from $\mathbb{B}_{r}$ into $M$ is homotopically equivalent to a disjoint union of $n ! \chi(M)$ copies of $\mathbb{T}^{n}$, and if $s \geq s_{0}$, then it is empty.

Proof. It follows easily from Lemma 2.3. Corollary 2.1 and the previous observations. 


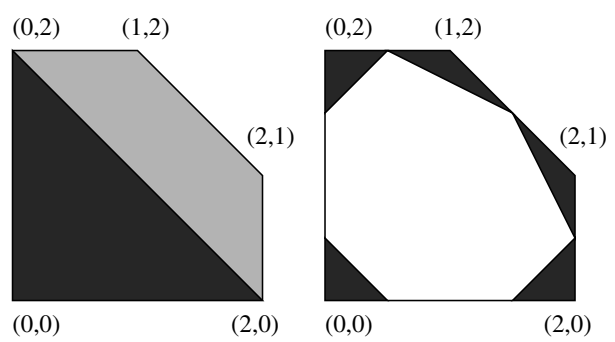

Figure 4. Polytope corresponding to the Delzant manifold $(M, \sigma)$ obtained by blowing up $\mathbb{S}_{r_{0}}^{2} \times \mathbb{S}_{r_{0}}^{2}$ with $r_{0}=1 / \sqrt{2}$. Observe that $\operatorname{Emb}_{(M, \sigma)}(\sqrt{2})=2$ (proof in left figure) and $\operatorname{Emb}_{(M, \sigma)}(1 / \sqrt{2})=10$ (proof in right figure). See Lemma 2.6.

This concludes the proof of Theorem 1.1 (and hence by construction the proof of Proposition 1.1).

\section{Remarks on the partially equivariant case of Theorem 1.1}

In this section we initiate a discussion on the topology of the space of partially equivariant symplectic embeddings and sketch some suggestions to answer a question in this direction. First the notion of $\Lambda$-equivariance $\left(\Lambda \in \operatorname{Aut}\left(\mathbb{T}^{n}\right)\right)$ in Section 1 has a natural extension: we say that an embedding from the $2 n$-ball $\mathbb{B}_{r}$ into the $2 n$ dimensional Delzant manifold $M$ is $\gamma$-equivariant with respect to a monomorphism $\gamma: \mathbb{T}^{n-k} \rightarrow \mathbb{T}^{n}, 1 \leq k \leq n-1$, if the following diagram commutes:

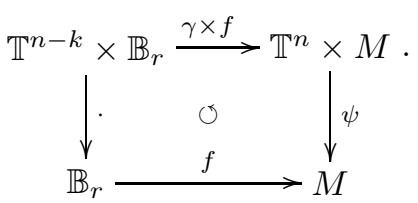

For example, $M^{\gamma}$ is the set of $p \in M$ such that $\psi(\gamma(t), p)=p$ for all $t \in \mathbb{T}^{n-k}$, and the rest of terminology is also analogous. This definition extends naturally to the case when $k=n$, in which the embeddings considered are purely symplectic, as well as to the case when $k=0$, in which the embeddings are fully equivariant, case which we treated previously in the paper. Unless otherwise specified we do not consider these two cases in the discussion that follows. The question we would like to address is the following:

Question 3.1. Is the space of $\gamma$-equivariant symplectic embeddings from $\mathbb{B}_{r}$ into $M$ homotopically equivalent to the space of purely symplectic embeddings from $\mathbb{B}_{r}^{2 k}$ into $M^{\gamma}$ up to certain reparametrization groups (as explained below)?

To analyze Question 3.1 first define $\widehat{\mathbb{B}}_{r}^{2 k}$ to be the embedded $2 k$-ball in $\mathbb{B}_{r}$, i.e. the set of points $\left(z_{1}, \ldots, z_{k}, 0\right)$ in $\mathbb{B}_{r}$ so that $\sum_{i=1}^{k}\left|z_{i}\right|^{2} \leq r^{2}$. The preimage under the momentum map of the $k$-face corresponding to $\gamma$ is the fixed point locus $M^{\gamma}$. Now consider any symplectic embedding $f: \widehat{\mathbb{B}}_{r}^{2 k} \rightarrow M^{\gamma}$. We want to find a canonical way to extend $f$ to an equivariant symplectic embedding $\operatorname{can}(f): \mathbb{B}_{r} \rightarrow M$ up to homotopy. 
Here is an attempt to construct can $(f)$ : near the image of $f$, we can apply the equivariant version of the Darboux-Weinstein's theorem in order to find a neighborhood of $\operatorname{Im}(f)$ in $M$ which is symplectomorphic to $\operatorname{Im}(f) \times \mathbb{B}^{2(n-k)}$, with the action of $\mathbb{T}^{n-k}$ given by the standard action on $\mathbb{B}^{2(n-k)}$, and the symplectic form coinciding with the product symplectic form. Note that the symplectic normal bundle to $M^{\gamma}$ is trivial over $\operatorname{Im}(f)$ because $\operatorname{Im}(f)$ is contractible, so a neighborhood of $\operatorname{Im}(f)$ looks like $\operatorname{Im}(f) \times \mathbb{B}^{2(n-k)}$ with a product symplectic form, and the action of $\mathbb{T}^{n-k}$ on it is conjugate to the standard one. Using this identification $M$ is described as a product, and we can define $\operatorname{can}(f)(z):=\left(f\left(z_{1}, \ldots, z_{k}\right), z_{k+1}, \ldots, z_{n}\right)$. This expression for $\operatorname{can}(f)$ is clearly symplectic and equivariant with respect to $\mathbb{T}^{n-k}$-actions on the last $n-k$ coordinates but is not canonical because the local symplectomorphism given by Darboux-Weinstein's theorem is not unique. We cannot expect it to always be the same independently of $f$, because it is not true that globally the normal bundle to $M^{\gamma}$ is symplectically trivial, it only becomes true over a neighborhood of $\operatorname{Im}(f)$. So this construction depends on choices of parameters.

Calling CAN the space of canonical embeddings $\operatorname{can}(f): \mathbb{B}_{r} \rightarrow \Delta^{M}$, where $f: \widehat{\mathbb{B}}_{r}^{2 k} \rightarrow M^{\gamma}$ is a symplectic embedding, observe that CAN is naturally identified with the space of purely symplectic embeddings from the standard $\mathbb{B}_{r}^{2 k}$ into $M^{\gamma}$, up to homotopy. The question then becomes whether any $\gamma$-equivariant symplectic embedding $f: \mathbb{B}_{r}^{2 k} \rightarrow M$ may be deformed through a continuous family of equivariant symplectic embeddings to an embedding in CAN.

Equivalently, we ask the question: is the natural map between the space of partially equivariant embeddings from $\mathbb{B}_{r}$ into $M$ and the space of symplectic embeddings from $\mathbb{B}_{r}^{2 k}$ into the fixed point set $M^{\gamma}$ (given by the restriction to the fixed ball $\left.\mathbb{B}_{r}^{2 k}\right)$ a fibration? Note that the construction of can $(f)$ would give a section of this fibration.

Conjecture 3.1. Question 3.1 has an affirmative answer.

Example 3. If $M=\mathbb{S}^{2} \times \mathbb{S}^{2}$ with a product symplectic form and product $\mathbb{T}^{2}$-action (this space has been carefully studied by Lalonde-Pinsonnault [14] and Anjos 2] among other authors), the fixed point locus of the second $\mathbb{S}^{1}$ factor is $\mathbb{S}^{2} \times\{a, b\}$, where $a, b$ are the fixed points of the action of $\mathbb{S}^{1}$ on $\mathbb{S}^{2}$. Now, given a symplectic embedding $f$ of the ball $\mathbb{B}^{2}$ into $\mathbb{S}^{2}$, it is easy to build an $\mathbb{S}^{1}$-equivariant embedding of $\mathbb{B}^{4}$ into $\mathbb{S}^{2} \times \mathbb{S}^{2}$ canonically by $\left(z_{1}, z_{2}\right) \mapsto\left(f\left(z_{1}\right), z_{2}\right)$, where $z_{2}$ is taken to be a coordinate centered at the fixed point $a$. In this case the normal bundle to the fixed point component $\mathbb{S}^{2} \times\{a\}$ is globally trivial.

The combination of purely symplectic results of Biran, Lalonde-Pinsonnault and others and an affirmative answer to Question 3.1 would give insight into the partially equivariant case in higher dimensions; for example McDuff showed that if $M$ is a symplectic 4-manifold with non-simple Seiberg-Witten type, then the space of symplectic embeddings from $\mathbb{B}_{r}$ into $M$ is path connected (which extends results of Biran). This is a consequence of the non-trivial result: any two cohomologous and deformation equivalent symplectic forms on $M$ are isotopic (proved in [17]). Examples are known in dimensions 6 and above of cohomologous symplectic forms that are deformation equivalent but not isotopic, so these techniques do not help to understand the topology of the space of symplectic embeddings from $\mathbb{B}_{r}$ into $M$. A 
positive answer to Question 3.1 would give the first non-trivial result in dimension 6.

Another way of trying to generalize Theorem 1.1 is to consider embeddings equivariant with respect to a complexity one action, that is, an action of $\mathbb{T}^{n-1}$ on $M^{2 n}$. This is a hopeful approach since a complete classification of complexity one actions has been recently achieved by Karshon and Tolman [15.

\section{ACKNOWLEDGMENTS}

The author is grateful to D. Auroux and V. Guillemin for discussions, and for hosting him at the M.I.T. regularly during the Fall and Spring semesters of 2003 and 2004. He thanks D. Auroux, J.J. Duistermaat, Y. Karshon and M. Pinsonnault for making comments on a preliminary version of this paper. Finally, the author is grateful to an anonymous referee for helpful suggestions that have shortened the proof in Section 2, as well as for his/her interesting comments on Section 3.

\section{REFERENCES}

[1] M. Atiyah, Convexity and commuting Hamiltonians. Bull. London Math. Soc., 1 (1982) 1-15. MR0642416 (83e:53037)

[2] S. Anjos, Homotopy type of symplectomorphism groups of $S^{2} \times S^{2}$. Geom. Topol. 6 (2002) 195-218. MR.1914568 (2003j:57052)

[3] P. Biran, Connectedness of spaces of symplectic embeddings, Int. Math. Res. Lett., 10 (1996) 487-491. MR1399413 (97g:58022)

[4] P. Biran, From symplectic packing to algebraic geometry and back, Proc. 3rd Europ. Congr. of Math., Barcelona 2, Prog. Math., Birkhauser (2001) 507-524. MR.1909952 (2003g:53150)

[5] P. Biran, Geometry of symplectic intersections, Proc. ICM Beijing III (2004) 1-3.

[6] A. Cannas da Silva, Lectures in symplectic geometry, Springer-Verlag, Berlin (2000). MR 1853077 (2002i:53105)

[7] T. Delzant, Periodic Hamiltonians and convex images of the momentum mapping, Bull. Soc. Math. France, 116 (1988) 315-339. MR0984900(90b:58069)

[8] V. Guillemin and S. Sternberg, Convexity properties of the momentum mapping, Invent. Math., 67 (1982) 491-513. MR0664117 (83m:58037)

[9] V. Guillemin and S. Sternberg, Symplectic techniques in physics, Cambr. Univ. Press (1984). MR0770935 (86f:58054)

[10] V. Guillemin, Moment maps and combinatorial invariants of Hamiltonian $\mathbb{T}^{n}$ spaces, Prog. Math., Birkhauser (1994). MR1301331 (96e:58064)

[11] V. Guillemin, V. Ginzburg and Y. Karshon, Moment maps, cobordisms and Hamiltonian group actions, Amer. Math. Soc. Monograph 98, (2002). MR1929136 (2003m:53149)

[12] M. Gromov, Pseudoholomorphic curves in symplectic geometry, Invent. Math., 82 (1985) 307-347. MR0809718 (87j:53053)

[13] M. Hirsch, Differential topology. Springer-Verlag 33 (1980).

[14] F. Lalonde and M. Pinsonnault, The topology of the space of symplectic balls in rational 4-manifolds, Duke Math. J. 122 (2004), no. 2, 347-397. MR.2053755(2005d:57038)

[15] Y. Karshon and S. Tolman, Centered complexity one Hamiltonian torus actions, Trans. Amer. Math. Soc. 353 (2001), no. 12, 4831-4861 (electronic). MR1852084 (2002g:53145)

[16] Y. Karshon and S. Tolman, The Gromov width of complex Grasmannians, Alg. and Geom. Topol. 5 paper 38, 911-922.

[17] D. McDuff, From deformation to isotopy, Topics in Symplectic 4-manifolds, Internat. Press, Cambridge, MA (1998). MR.1635697(99j:57025)

[18] D. McDuff, The structure of rational and ruled symplectic 4-manifolds, J. Amer. Math. Soc. 3(3) (1990) 679-712. MR 1049697 (91k:58042)

[19] A. Pelayo, Toric symplectic ball packing, To appear in Topol. Appl..

Department of Mathematics, University of Michigan, 2074 East Hall, 530 Church Street, Ann Arbor, Michigan 48109-1043

E-mail address: apelayo@umich.edu 